Supplement of Hydrol. Earth Syst. Sci., 24, 3331-3359, 2020

https://doi.org/10.5194/hess-24-3331-2020-supplement

(c) Author(s) 2020. This work is distributed under

the Creative Commons Attribution 4.0 License.

(c) (1)
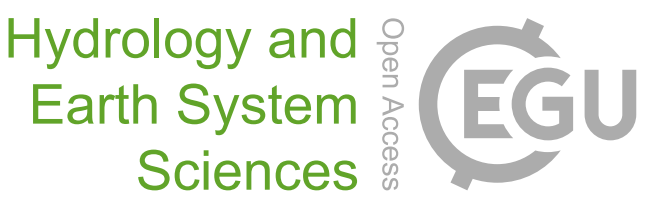

Supplement of

\title{
Using altimetry observations combined with GRACE to select parameter sets of a hydrological model in a data-scarce region
}

Petra Hulsman et al.

Correspondence to: Petra Hulsman (p.hulsman@tudelft.nl)

The copyright of individual parts of the supplement might differ from the CC BY 4.0 License. 


\section{Supplements}

\section{Background on the altimetry data from LEGOS}

The altimetry data obtained from LEGOS come from the acquisitions of ENVISAT and Jason-2 radar altimetry missions on their nominal orbit (03/2002-10/2010 and 06/2008-10/2016 respectively). All the parameters necessary to estimate water levels (Crétaux et al., 2017) are contained in the Geophysical Data Records (GDR) made available by the space agencies. These data were obtained from Centre de Topographie des Océans et de l'Hydrosphère (CTOH - http://ctoh.legos.obs-mip.fr). Ranges used to derive altimeter heights are those processed using OCOG/Ice retracking algorithm (Wingham et al., 1986). Previous studies showed that altimeter heights derived using this retracking algorithm are more suitable for hydrological studies in terms of accuracy of water levels and availability of the data (Frappart et al., 2006; Santos da Silva et al., 2010; Sulistioadi et al., 2015) among the commonly available retracked data present in the GDRs.

The Multi-mission Altimetry Processing Software (MAPS) was used to visualize and process the altimetry data in order to obtain the virtual stations (VS) at the cross-sections between the altimeter ground tracks and the rivers

(Frappart et al., 2015; Normandin et al., 2018). Data processing is composed of three main steps: (i) a coarse delineation of the VS using Google Earth; (ii) a refined selection of the valid altimetry data based on visual inspection; and (iii) the computation of the time series of water level. The altimetry-based water level is computed for each cycle using the median of the selected altimetry heights, along with their respective deviation (i.e., mean absolute deviation). This process is repeated each cycle to construct the water level time series at the virtual stations; see Frappart et al., 2015; Normandin et al., 2018 for more details.

\section{Literature}

Crétaux, J.-F., Nielsen, K., Frappart, F., Papa, F., Calmant, S., and Benveniste, J.: Hydrological applications of satellite altimetry: rivers, lakes, man-made reservoirs, inundated areas, in: Satellite Altimetry Over Oceans and Land Surfaces; Earth Observation of Global Changes, edited by: Stammer, D., and Cazenave, A., CRC Press, Boca Raton, FL, USA, 459-504, 2017.

Frappart, F., Calmant, S., Cauhopé, M., Seyler, F., and Cazenave, A.: Preliminary results of ENVISAT RA-2derived water levels validation over the Amazon basin, Remote Sensing of Environment, 100, 252-264, https://doi.org/10.1016/j.rse.2005.10.027, 2006.

30 Frappart, F., Papa, F., Marieu, V., Malbeteau, Y., Jordy, F., Calmant, S., Durand, F., and Bala, S.: Preliminary Assessment of SARAL/AltiKa Observations over the Ganges-Brahmaputra and Irrawaddy Rivers, Marine Geodesy, 38, 568-580, 10.1080/01490419.2014.990591, 2015.

Normandin, C., Frappart, F., Telly Diepkilé, A., Marieu, V., Mougin, E., Blarel, F., Lubac, B., Braquet, N., and Ba, A.: Evolution of the Performances of Radar Altimetry Missions from ERS-2 to Sentinel-3A over the Inner $35 \quad$ Niger Delta, 833 pp., 2018.

Santos da Silva, J., Calmant, S., Seyler, F., Rotunno Filho, O. C., Cochonneau, G., and Mansur, W. J.: Water levels in the Amazon basin derived from the ERS 2 and ENVISAT radar altimetry missions, Remote Sensing of Environment, 114, 2160-2181, https://doi.org/10.1016/j.rse.2010.04.020, 2010.

Sulistioadi, Y. B., Tseng, K. H., Shum, C. K., Hidayat, H., Sumaryono, M., Suhardiman, A., Setiawan, F., and Sunarso, S.: Satellite radar altimetry for monitoring small rivers and lakes in Indonesia, Hydrol. Earth Syst. Sci., 19, 341-359, 10.5194/hess-19-341-2015, 2015.

Wingham, D., Rapley, C., and H D, G.: New Techniques in Satellite Altimeter Tracking Systems, 1986. 


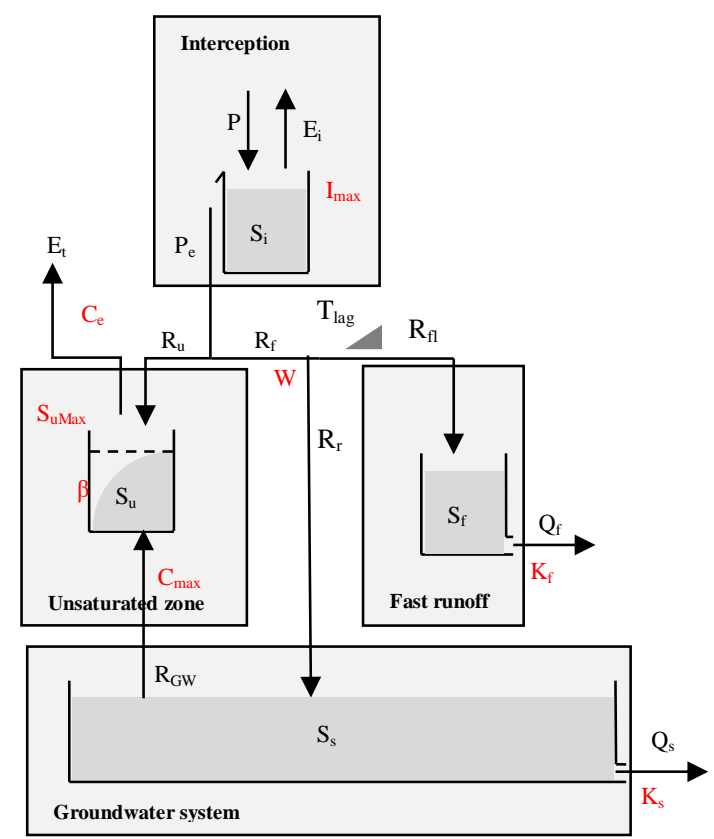

Figure S1: Model structure. Parameters are marked in red, storages and fluxed in black. Symbol explanation: Fluxes $\left[\mathrm{mm} \mathrm{d}^{-1}\right]$ : precipitation $(P)$, effective precipitation $\left(P_{\mathrm{e}}\right)$, potential evaporation $\left(E_{\mathrm{p}}\right)$, interception evaporation $\left(E_{\mathrm{i}}\right)$, plant transpiration $\left(E_{\mathrm{t}}\right)$, infiltration into the unsaturated zone $\left(R_{\mathrm{u}}\right)$, drainage to fast runoff component $\left(R_{\mathrm{f}}\right)$, delayed fast runoff $\left(R_{\mathrm{f}}\right)$, groundwater recharge $\left(R_{\mathrm{r}}\right)$, upwelling groundwater $\left(\boldsymbol{R}_{\mathrm{GW}}\right)$, fast runoff $\left(Q_{\mathrm{f}}\right)$, groundwater/slow runoff $\left(Q_{\mathrm{s}}\right)$, total runoff $\left(Q_{\mathrm{m}}\right)$. Storages [mm]: storage in interception reservoir $\left(S_{\mathrm{i}}\right)$, storage in unsaturated root zone $\left(S_{\mathrm{u}}\right)$, storage in groundwater/slow reservoir $\left(S_{\mathrm{s}}\right)$, storage in fast reservoir $\left(S_{\mathrm{f}}\right)$. Parameters: interception capacity $\left(I_{\max }\right)$ [mm], maximum upwelling groundwater $\left(C_{\max }\right)\left[\mathrm{mm} \mathrm{d}^{-1}\right]$, maximum root zone storage capacity $\left(S_{\text {umax }}\right)[\mathrm{mm}]$, splitter $(W)[-]$, shape parameter $(\beta)$ [-], transpiration coefficient $\left(C_{e}\right)$ [-], time lag $\left(T_{\text {lag }}\right)$ [d], reservoir time scales [d] of fast $\left(K_{\mathrm{f}}\right)$ and slow $\left(K_{s}\right)$ reservoirs. 
Table S1: Model parameter values and ranges. See Figure S1 for the parameter explanation and Table S2 for the parameter constrains applied during the random parameter generation.

\begin{tabular}{|c|c|c|c|c|}
\hline Landscape class & Parameter & $\min$ & $\max$ & Unit \\
\hline \multirow[t]{2}{*}{ Entire catchment } & $\mathrm{K}_{\mathrm{s}}$ & 100 & 100 & $\mathrm{~d}$ \\
\hline & $\mathrm{C}_{\mathrm{e}}$ & 0.5 & 0.5 & - \\
\hline \multirow[t]{4}{*}{ Plateau/Terrace } & $\mathrm{I}_{\max }$ & 0 & 2 & $\mathrm{~mm} \mathrm{~d}^{-1}$ \\
\hline & $\mathrm{S}_{\mathrm{umax}}$ & 200 & 2000 & $\mathrm{~mm}$ \\
\hline & $\mathrm{K}_{\mathrm{f}}$ & 10 & 12 & d \\
\hline & W & 0.1 & 0.5 & - \\
\hline \multirow[t]{6}{*}{ Hillslope } & $\mathrm{I}_{\max }$ & 0 & 2 & $\mathrm{~mm} \mathrm{~d}^{-1}$ \\
\hline & $S_{\text {umax }}$ & 200 & 2000 & $\mathrm{~mm}$ \\
\hline & $\beta$ & 0 & 2 & - \\
\hline & $\mathrm{T}_{\text {lag }}$ & 1 & 5 & d \\
\hline & $\mathrm{K}_{\mathrm{f}}$ & 10 & 12 & d \\
\hline & W & 0.1 & 0.5 & - \\
\hline \multirow[t]{5}{*}{ Wetland } & $\mathrm{I}_{\max }$ & 0 & 2 & $\mathrm{~mm} \mathrm{~d}^{-1}$ \\
\hline & $S_{\text {umax }}$ & 200 & 2000 & $\mathrm{~mm}$ \\
\hline & $\mathrm{K}_{\mathrm{f}}$ & 10 & 12 & d \\
\hline & W & 0.1 & 0.5 & - \\
\hline & $\mathrm{C}_{\max }$ & 0.1 & 2 & $\mathrm{~mm} \mathrm{~d}^{-1}$ \\
\hline \multirow[t]{4}{*}{ River profile } & $\mathrm{v}$ & 0.01 & 5.0 & $\mathrm{~m} \mathrm{~s}^{-1}$ \\
\hline & $\mathrm{k}$ & 5 & 45 & $\mathrm{~m}^{1 / 3} \mathrm{~s}^{-1}$ \\
\hline & $\mathrm{a}$ & 0.1 & 800 & $\mathrm{~m}^{3} \mathrm{~s}^{-1}$ \\
\hline & $\mathrm{b}$ & 1 & 3 & - \\
\hline
\end{tabular}

Table S2: Parameter constrains. See Figure S1 for the parameter explanation.

\begin{tabular}{l|c} 
Parameter & Constrain \\
\hline Maximum root zone storage capacity & $\mathrm{S}_{\text {umax,hillslope }}>\mathrm{S}_{\text {umax,plateau/terrace }}$ \\
Maximum interception & $\mathrm{S}_{\text {umax,hillslope }}>\mathrm{S}_{\text {umax,wetland }}$ \\
& $\mathrm{I}_{\text {max,hillslope }}>\mathrm{I}_{\text {max,plateau/terrace }}$ \\
Splitter for groundwater percolation & $\mathrm{I}_{\text {max,hillslope }}>\mathrm{I}_{\text {max,wetland }}$ \\
& $\mathrm{W}_{\text {hillslope }}>W_{\text {plateau/terrace }}$
\end{tabular}




\section{Characteristics of the virtual stations}

Table S3: Characteristics of the virtual stations in the Luangwa River basin for which remotely sensed river water levels are available: station ID, coordinates $(X, Y)$, river slope $(i)$, river width $(B)$, river bank slopes $\left(i_{1}\right.$ and $\left.i_{2}\right)$, upstream catchment area, acquisition date of the image in Google Earth used to extract the river geometry information, and discharge at Luangwa Bridge gauge station (basin outlet; absolute values and relative to the maximum discharge); in the absence of discharge data on the acquisition dates, the long-term mean daily values for the entire time period available were used.

\begin{tabular}{|c|c|c|c|c|c|c|c|c|c|}
\hline VS & $\mathbf{X}$ & $\mathbf{Y}$ & i [ [-] & $\mathbf{B}[\mathbf{m}]$ & $\mathbf{i}_{1}[-]$ & $\mathbf{i}_{2}[-]$ & $\mathbf{A}\left[\mathbf{m}^{2}\right]$ & $\begin{array}{c}\text { Acquisition } \\
\text { date }\end{array}$ & $\begin{array}{c}\mathbf{Q}_{\text {absolute }}\left[\mathrm{m}^{3} \mathbf{s}^{-1}\right] \\
\left(\mathbf{Q}_{\text {relative }}[\%]\right)\end{array}$ \\
\hline 1 & $30.2823^{\circ}$ & $-14.8664^{\circ}$ & 0.00049 & 324 & 36 & 29 & 10211995771 & $13-9-2010$ & $68(1 \%)$ \\
\hline 2 & $30.0864^{\circ}$ & $-14.366^{\circ}$ & 0.00062 & 7 & 17 & 83 & 14859805930 & $13-10-2013$ & $65(1 \%)$ \\
\hline 3 & $32.1715^{\circ}$ & $-12.4123^{\circ}$ & 0.00019 & 3 & 19 & 42 & 44337218380 & $17-12-2013$ & $211(4 \%)$ \\
\hline 4 & $31.1868^{\circ}$ & $-13.5927^{\circ}$ & 0.00020 & 129 & 42 & 8 & 87227195673 & $5-6-2013$ & $160(3 \%)$ \\
\hline 5 & $31.6984^{\circ}$ & $-13.2039^{\circ}$ & 0.00020 & 185 & 31 & 20 & 78090945429 & $20-9-2013$ & $60(1 \%)$ \\
\hline 6 & $32.2998^{\circ}$ & $-12.2007^{\circ}$ & 0.00039 & 170 & 30 & 17 & 40935244516 & $13-6-2013$ & $146(3 \%)$ \\
\hline 7 & $32.2805^{\circ}$ & $-12.1157^{\circ}$ & 0.00030 & 78 & 38 & 77 & 40747298483 & $13-6-2013$ & $146(3 \%)$ \\
\hline 8 & $32.831^{\circ}$ & $-11.3674^{\circ}$ & 0.00031 & 10 & 48 & 21 & 21066101487 & 26-9-2013 & $97(2 \%)$ \\
\hline 9 & $30.2704^{\circ}$ & $-14.8809^{\circ}$ & 0.00017 & 99 & 8 & 5 & 102140213550 & 14-11-2009 & $30(1 \%)$ \\
\hline 10 & $31.78405^{\circ}$ & $-13.0995^{\circ}$ & 0.00029 & 100 & 26 & 20 & 77559639645 & $26-7-2013$ & $89(2 \%)$ \\
\hline 11 & $31.71099^{\circ}$ & $-13.1943^{\circ}$ & 0.00020 & 54 & 34 & 30 & 78051272962 & $20-9-2013$ & $60(1 \%)$ \\
\hline 12 & $30.2740^{\circ}$ & $-14.8763^{\circ}$ & 0.00017 & 82 & 8 & 15 & 102135928406 & 14-11-2009 & $30(1 \%)$ \\
\hline 13 & $32.15843^{\circ}$ & $-12.412^{\circ}$ & 0.00019 & 87 & 43 & 30 & 44340963341 & $17-12-2013$ & $211(4 \%)$ \\
\hline 14 & $32.15989^{\circ}$ & $-12.4127^{\circ}$ & 0.00019 & 128 & 83 & 19 & 44339840479 & $13-6-2013$ & $146(3 \%)$ \\
\hline 15 & $30.2740^{\circ}$ & $-14.8763^{\circ}$ & 0.00017 & 82 & 8 & 15 & 102139379771 & $13-6-2013$ & $146(3 \%)$ \\
\hline 16 & $32.16056^{\circ}$ & $-12.4125^{\circ}$ & 0.00019 & 128 & 83 & 19 & 44339840479 & $17-12-2013$ & $211(4 \%)$ \\
\hline 17 & $31.80001^{\circ}$ & $-13.0909^{\circ}$ & 0.00029 & 86 & 21 & 83 & 77553414963 & $13-6-2013$ & $146(3 \%)$ \\
\hline 18 & $30.61577^{\circ}$ & $-14.1852^{\circ}$ & 0.00051 & 227 & 24 & 20 & 96231647197 & $20-9-2014$ & $60(1 \%)$ \\
\hline Outlet & $30.21491^{\circ}$ & $-14.96678^{\circ}$ & 0.00037 & 149 & 8.62 & 10.10 & 154325857000 & $26-7-2016$ & $89(2 \%)$ \\
\hline
\end{tabular}
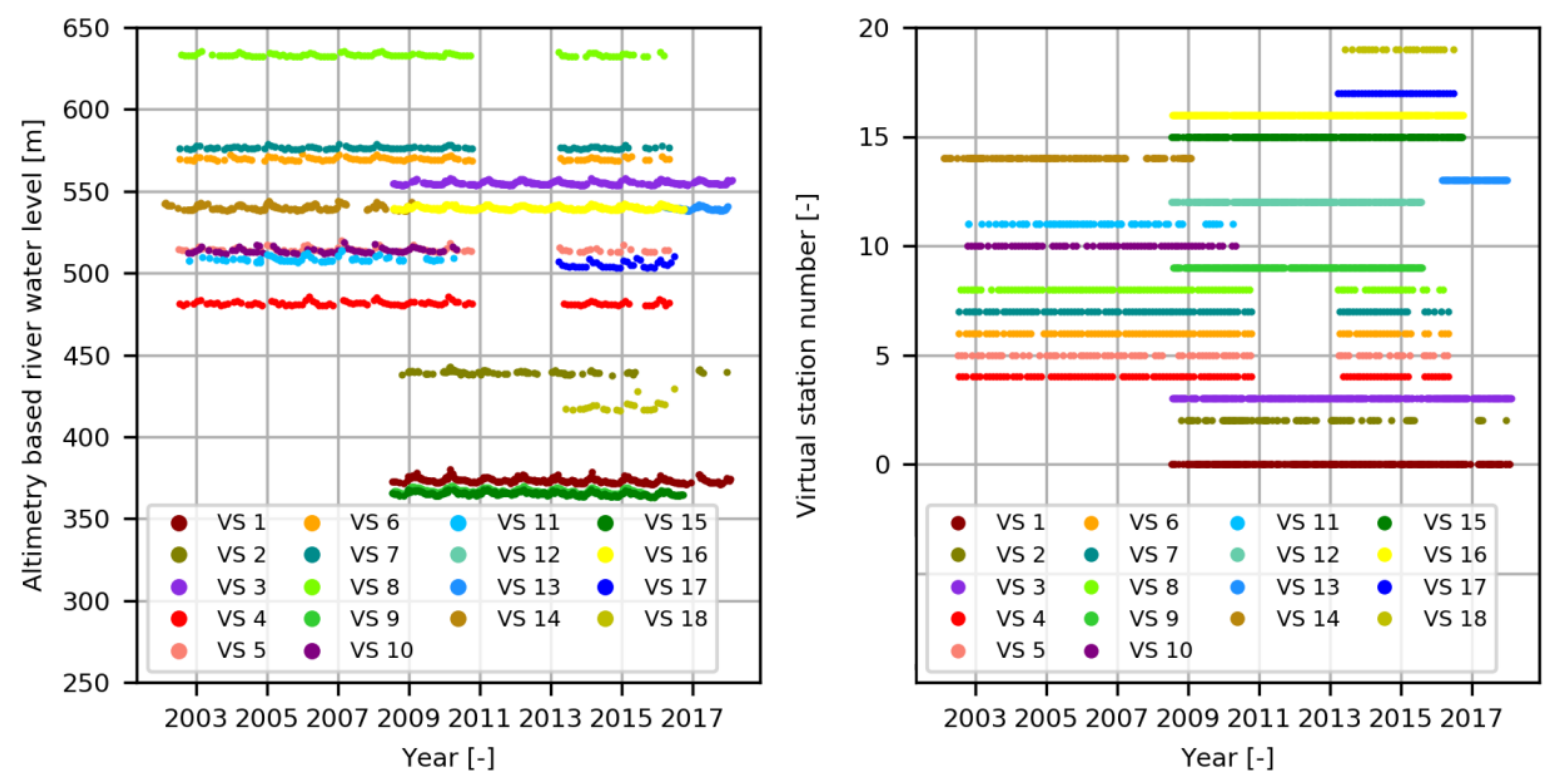

Figure S2: Visualisation of the altimetry time series relative to a reference ellipsoid (left) and altimetry data availability (right) for all virtual stations used in this study. The colours for the individual stations correspond with those in Figure 1 and 3. 

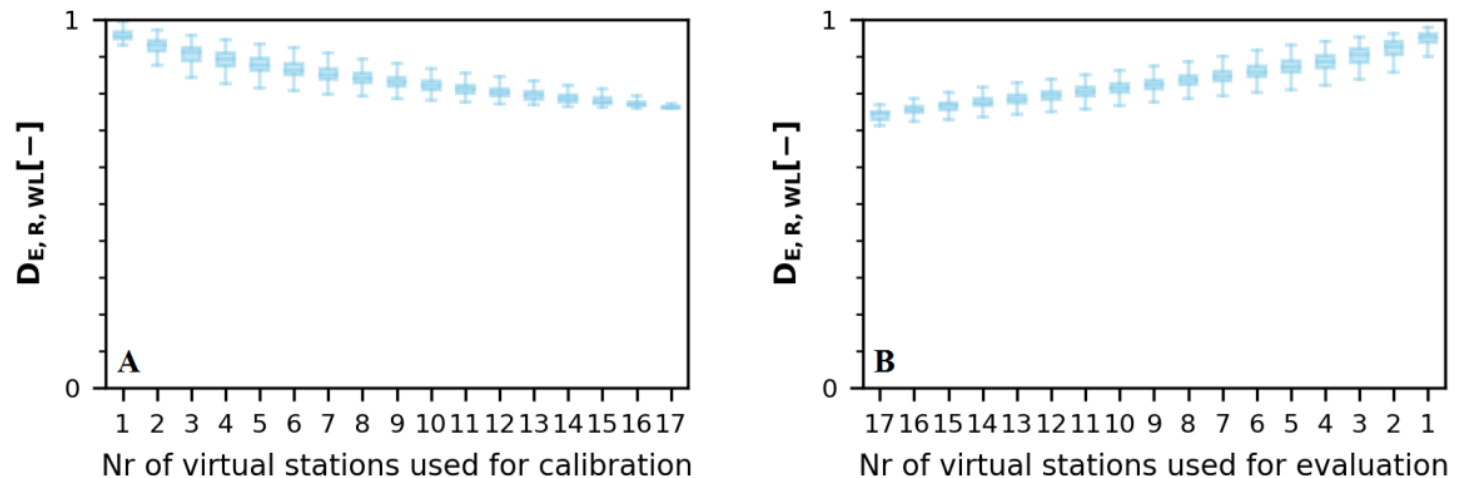

Figure S3: Influence of the number of virtual stations used for A) model calibration and B) evaluation on the model performance $D_{\mathrm{E}, \mathrm{R}, \mathrm{WL}}$ applying Altimetry Strategy 1.
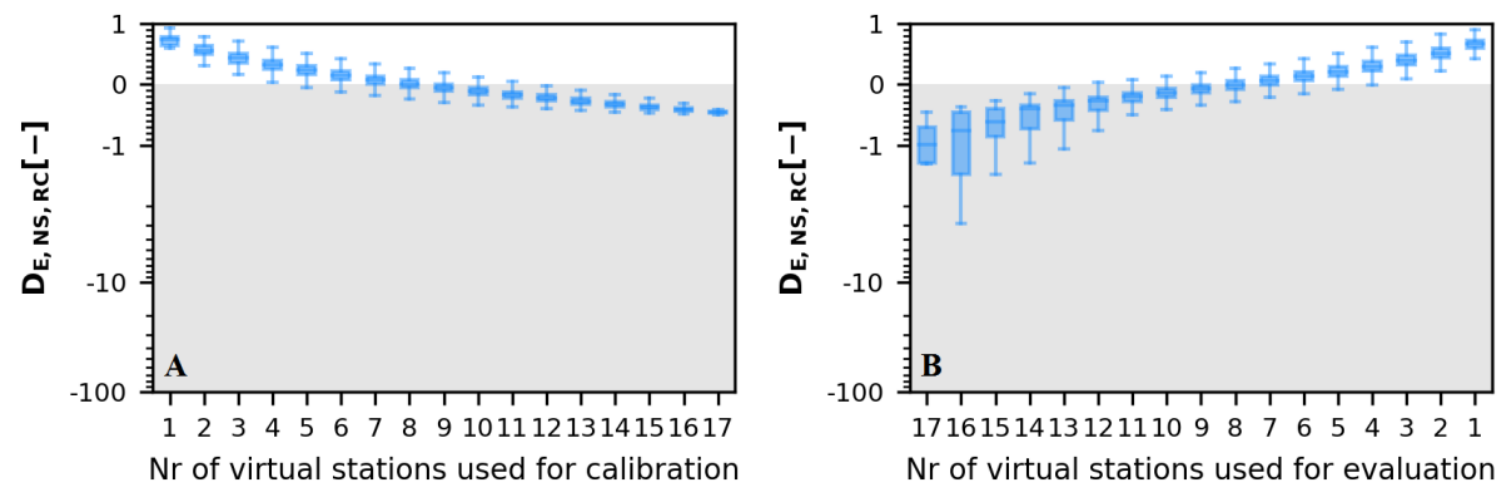

Figure S4: Influence of the number of virtual stations used for A) model calibration and B) evaluation on the model performance $D_{\mathrm{E}, \mathrm{NS}, \mathrm{RC}}$ applying Altimetry Strategy 2. 


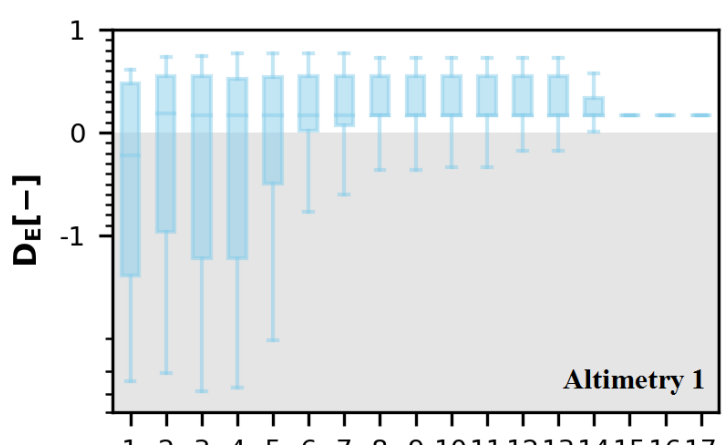

$\begin{array}{llllllllll}1 & 2 & 3 & 4 & 5 & 6 & 7 & 8 & 9 & 1011121314151617\end{array}$

$\mathrm{Nr}$ of virtual stations used for calibration

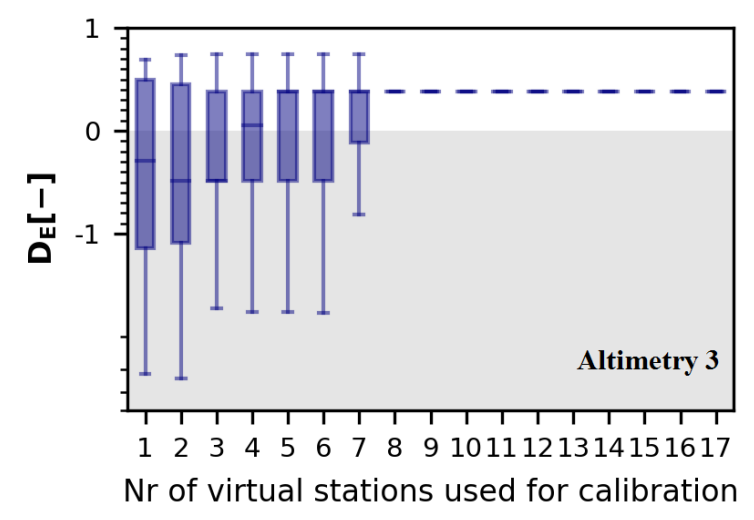

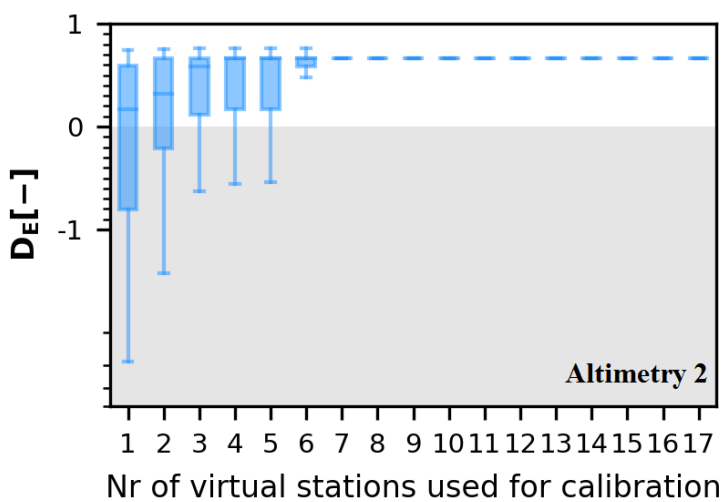

Figure S5: Influence of the number of virtual stations used for model calibration on the model performance $D_{E}$ with respect to discharge 


\section{Model performance with respect to discharge}

Table S4: Model performance with respect to each flow signature separately $\left(E_{\mathrm{NS}, \mathrm{Q}}, E_{\mathrm{NS}, \log Q}, E_{\mathrm{NS}, \mathrm{FDC}}, E_{\mathrm{NS}, \operatorname{logFDC}}\right.$, $\left.E_{\mathrm{R}, \mathrm{RCdry}}, E_{\mathrm{R}, \mathrm{RCwet}}, E_{\mathrm{NS}, \mathrm{AC}}, E_{\mathrm{R}, \mathrm{RLD}}\right)$ for each parameter identification strategy.

\begin{tabular}{|c|c|c|c|c|c|c|c|c|}
\hline & $E_{\mathrm{NS}, \mathrm{Q}}$ & $E_{\mathrm{NS}, \log Q}$ & $\boldsymbol{E}_{\mathrm{NS}, \mathrm{FDC}}$ & $E_{\mathrm{NS}, \log F \mathrm{C}}$ & $E_{\mathrm{R}, \mathrm{RCdry}}$ & $E_{\mathrm{R}, \mathrm{RCwet}}$ & $E_{\mathrm{NS}, \mathrm{AC}}$ & $E_{\mathrm{R}, \mathrm{RLD}}$ \\
\hline Discharge & $\begin{array}{c}0.78 \\
(0.68-0.76)\end{array}$ & $\begin{array}{c}0.56 \\
(0.61-0.78)\end{array}$ & $\begin{array}{c}0.95 \\
(0.81-0.97)\end{array}$ & $\begin{array}{c}0.88 \\
(0.80-0.97)\end{array}$ & $\begin{array}{c}0.08 \\
(0.11-0.97)\end{array}$ & $\begin{array}{c}0.67 \\
(0.61-0.84)\end{array}$ & $\begin{array}{c}0.94 \\
(0.89-0.97)\end{array}$ & $\begin{array}{c}0.73 \\
(0.29-0.75)\end{array}$ \\
\hline $\begin{array}{l}\text { Seasonal } \\
\text { water storage }\end{array}$ & $\begin{array}{c}-1.38 \\
(-2.2--0.05)\end{array}$ & $\begin{array}{c}0.09 \\
(0.11-0.73)\end{array}$ & $\begin{array}{c}-0.80 \\
(-1.6-0.40)\end{array}$ & $\begin{array}{c}0.25 \\
(0.27-0.93)\end{array}$ & $\begin{array}{c}0.52 \\
(0.45-0.97)\end{array}$ & $\begin{array}{c}0.27 \\
(0.24-0.36)\end{array}$ & $\begin{array}{c}0.98 \\
(0.97-0.99)\end{array}$ & $\begin{array}{c}0.61 \\
(0.14-0.79)\end{array}$ \\
\hline $\begin{array}{l}\text { Altimetry } \\
\text { Strategy } 1\end{array}$ & $\begin{array}{c}0.65 \\
(-2.9-0.08)\end{array}$ & $\begin{array}{c}0.69 \\
(0.20-0.69)\end{array}$ & $\begin{array}{c}0.93 \\
(-2.0-0.68)\end{array}$ & $\begin{array}{c}0.85 \\
(0.37-0.89)\end{array}$ & $\begin{array}{c}0.87 \\
(0.42-0.81)\end{array}$ & $\begin{array}{c}0.64 \\
(0.22-0.44)\end{array}$ & $\begin{array}{c}0.92 \\
(0.97-0.99)\end{array}$ & $\begin{array}{c}0.15 \\
(0.10-0.46)\end{array}$ \\
\hline $\begin{array}{l}\text { Altimetry } \\
\text { Strategy } 2\end{array}$ & $\begin{array}{c}-0.31 \\
(-2.5-0.27)\end{array}$ & $\begin{array}{c}0.26 \\
(-0.12-0.66)\end{array}$ & $\begin{array}{c}0.10 \\
(-1.9-0.73)\end{array}$ & $\begin{array}{c}0.41 \\
(0.05-0.88)\end{array}$ & $\begin{array}{c}0.55 \\
(0.38-0.89)\end{array}$ & $\begin{array}{c}0.34 \\
(0.23-0.46)\end{array}$ & $\begin{array}{c}0.99 \\
(0.97-0.99)\end{array}$ & $\begin{array}{c}0.51 \\
(0.11-0.70)\end{array}$ \\
\hline $\begin{array}{l}\text { Altimetry } \\
\text { Strategy } 3\end{array}$ & $\begin{array}{c}0.61 \\
(-0.30-0.50)\end{array}$ & $\begin{array}{c}0.70 \\
(0.45-0.78)\end{array}$ & $\begin{array}{c}0.95 \\
(0.16-0.88)\end{array}$ & $\begin{array}{c}0.91 \\
(0.65-0.99)\end{array}$ & $\begin{array}{c}0.93 \\
(0.54-0.98)\end{array}$ & $\begin{array}{c}0.60 \\
(0.34-0.54)\end{array}$ & $\begin{array}{c}0.99 \\
(0.98-0.99)\end{array}$ & $\begin{array}{c}0.52 \\
(0.29-0.79)\end{array}$ \\
\hline $\begin{array}{l}\text { Water level } \\
\text { Strategy } 1\end{array}$ & $\begin{array}{c}0.65 \\
(-0.49-0.60)\end{array}$ & $\begin{array}{c}0.74 \\
(0.37-0.75)\end{array}$ & $\begin{array}{c}0.97 \\
(0.13-0.95)\end{array}$ & $\begin{array}{c}0.94 \\
(0.593-0.98)\end{array}$ & $\begin{array}{c}0.83 \\
(0.52-0.98)\end{array}$ & $\begin{array}{c}0.64 \\
(0.34-0.60)\end{array}$ & $\begin{array}{c}0.98 \\
(0.98-0.99)\end{array}$ & $\begin{array}{c}0.76 \\
(0.12-0.75)\end{array}$ \\
\hline $\begin{array}{l}\text { Water level } \\
\text { Strategy } 2\end{array}$ & $\begin{array}{c}0.14 \\
(-1.2-0.50)\end{array}$ & $\begin{array}{c}0.78 \\
(0.53-0.77)\end{array}$ & $\begin{array}{c}0.57 \\
(-0.62-0.91)\end{array}$ & $\begin{array}{c}0.98 \\
(0.73-0.98)\end{array}$ & $\begin{array}{c}0.64 \\
(0.66-0.99)\end{array}$ & $\begin{array}{c}0.40 \\
(0.28-0.55)\end{array}$ & $\begin{array}{c}0.98 \\
(0.98-0.99)\end{array}$ & $\begin{array}{c}0.57 \\
(0.26-0.77)\end{array}$ \\
\hline
\end{tabular}


6 Model performance with respect to river water level
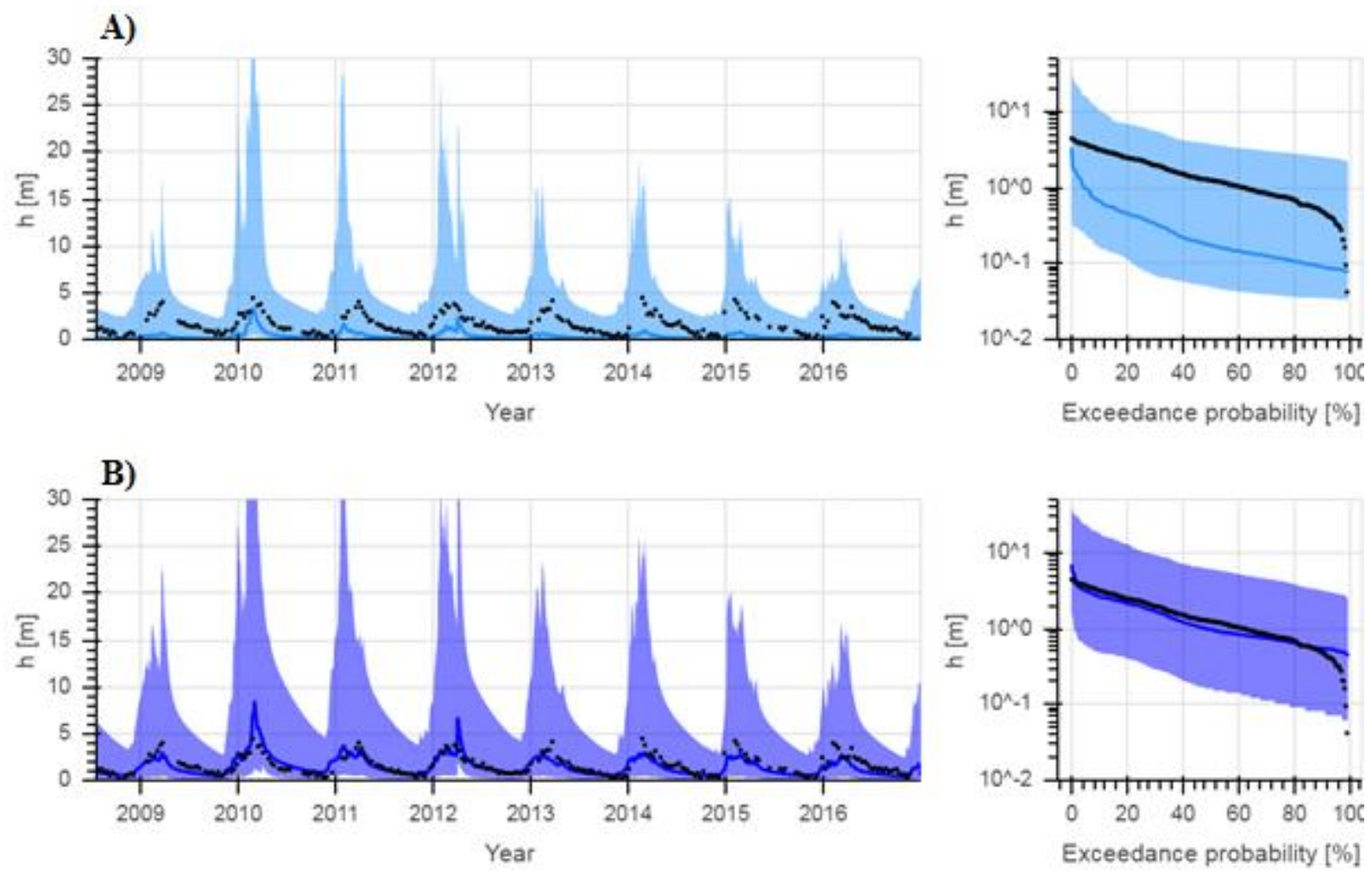

Figure S6: Range of model solutions for Virtual Station 4 (see Figure 1 for its location). The left panel shows the time series and the right panel the exceedance probability graph of the recorded (black) and modelled water level: the line indicates the solution with the highest calibration objective function and the shaded area the envelope of the solutions retained as feasible. Solutions retained as feasible based on altimetry observations using all virtual stations within the basin and A) calibrated rating curves for the discharge - water level conversion (Altimetry Strategy 2) or B) the Strickler-Manning equation with cross-section information retrieved from Google Earth (Altimetry Strategy 3). 
7 Model performance comparison
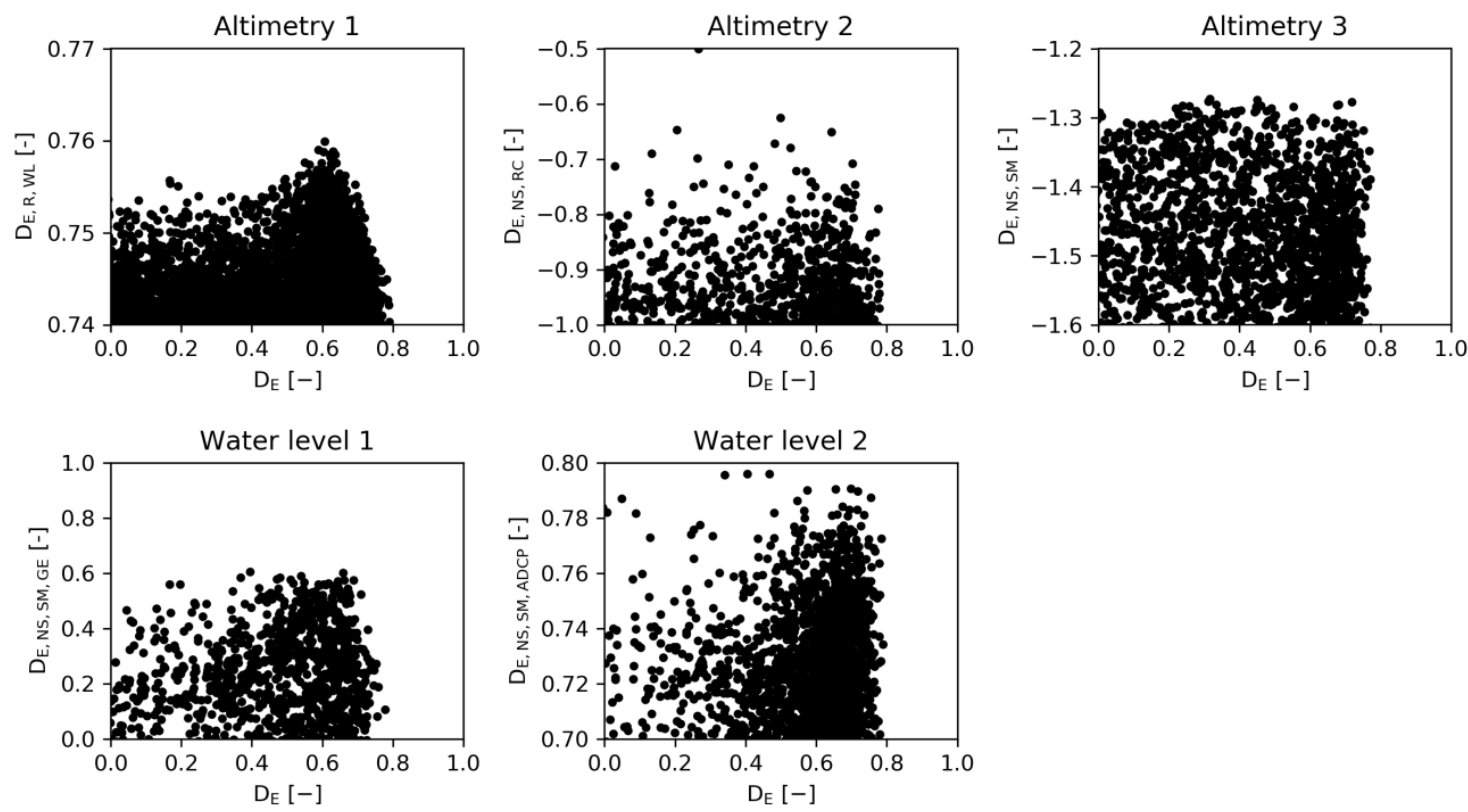

Figure S7: Model performance with respect to discharge (horizontal axes) vs. model performance with respect to (satellite based) river water level (vertical axes) for each calibration strategy

8 Influence of the total water storage on the model performance

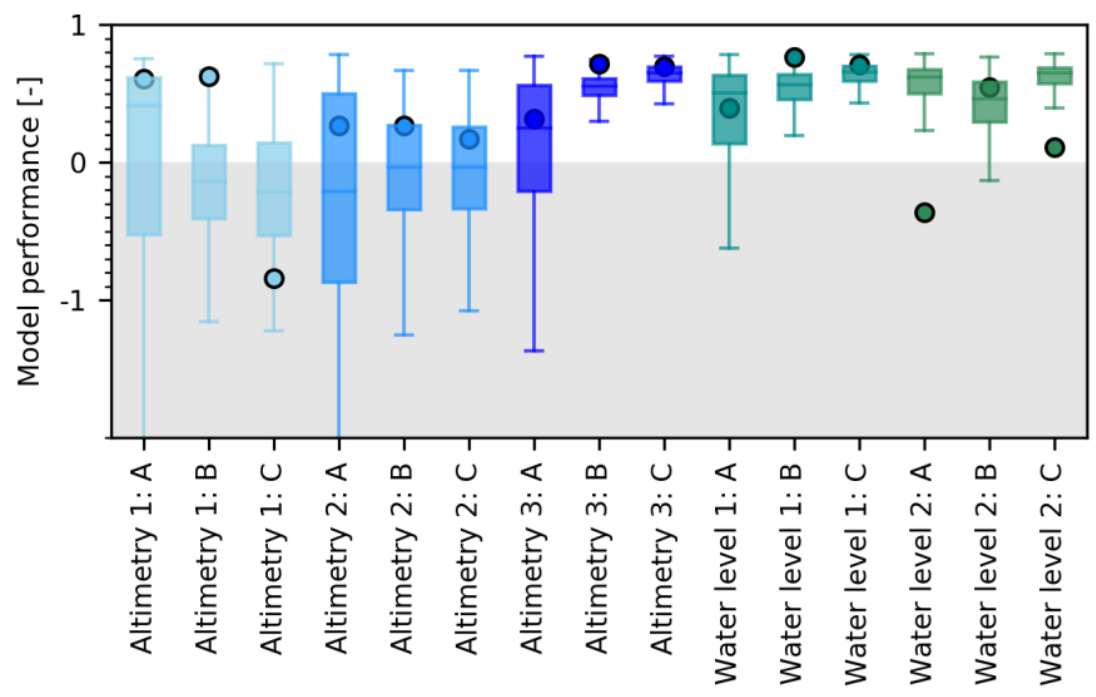

Figure S8: Model performance with respect to discharge for each calibration strategy. Parameter sets were selected based on A) (satellite based) river water level only, B) first GRACE, then (satellite based) river water level, and C) first (satellite based) river water level, then GRACE 